\title{
CT Evaluation of Injuries to Temporal Bone in Case of Trauma, Prospective Study
}

\author{
Anand M Rahalkar ${ }^{1}$, Manali Rahalkar ${ }^{2}$, Mukund Rahalkar ${ }^{3}$ \\ ${ }^{1}$ Associate Professor, Department of Radiology, Sassoon General Hospitals and BJ Medical College, Pune 400001, ${ }^{2}$ Associate \\ Professor, Department of Radiology, Sassoon General Hospitals and BJ Medical College, Pune 400001, ${ }^{3}$ Ex Professor, \\ Department of Radiology, Sassoon General Hospitals and BJ Medical College, Pune 400001, India
}

Corresponding author: Dr. Manali A Rahalkar, 721/2B, Vikasnagar, Navi Peth, Pune 411030, India

DOI: http://dx.doi.org/10.21276/ijcmsr.2019.4.3.28

How to cite this article: Anand M Rahalkar, Manali Rahalkar, Mukund Rahalkar. CT evaluation of injuries to temporal bone in case of trauma, prospective study. International Journal of Contemporary Medicine Surgery and Radiology. 2019;4(3):C124-C127.

\section{A B S T R A C T}

Introduction: HRCT temporal bone is the key to assessment of trauma of temporal bone. The main purpose of this study was to assess the injuries occurring to temporal bone in case of trauma.

Material and Methods: Institutional Ethical Committee Approval was taken prior to commencement of the present study. Written and informed consent was taken from all patients for including them into this study.

Patients were selected on the basis of inclusion and exclusion criteria. A case record proforma was prepared for each patient. A total of 50 patients referred to Radio diagnosis department between February 2013 and February 2014 were selected with history of trauma and ear bleed or swelling or hearing loss following trauma. All patients were scanned on Siemens 64 slice CT scanner.

Results: Ossicular injuries are not so common in cases of trauma. Fractures and hemorrhage in the middle ear was more common. We got 5 cases of ossicular injury after assessing 50 cases of trauma to ear. The incus was more commonly involved in trauma than the malleus and stapes.

Conclusion: Out of the 3 ossicles in the middle ear, the incus in the most commonly involved ossicle. This is thought to be due to firm attachment of the malleus to the tympanic membrane and stapes to the oval window.

Keywords: HRCT temporal bone, Longitudinal and transverse fractures, Ossicular injuries

\section{INTRODUCTION}

The fractures occurring to the temporal bone can either be longitudinal or transverse. Oblique fractures are also described. The mechanism of injury leading to longitudinal fracture is a temporal or parietal trauma. Transverse fractures result from frontal or occipital impact. ${ }^{1}$ Oblique fractures are the most common type of fractures. ${ }^{1,2,3}$

There are different types of injures to ear ossicles including ossicular chain dislocation. Typically five different types of ossicular disclocations are described in literature, incudostapedial joint dislocation; malleoincudal joint dislocation; dislocation of the incus; dislocation of the malleoincudal complex and stapediovestibular dislocation. ${ }^{1}$ Conductive deafness can occur due to injury to the ossicles following trauma and is more common with longitudinal fracture. Sensorineural deafness occur more commonly with transverse fractures due to injury to the inner ear structures or cochlear nerve. ${ }^{1}$ Sensorineural deafness can also occur due to cochlear concussion but is rare. ${ }^{1}$ Complications can be chronic otitis media or development of choleteatoma.

Study aimed to assess fractures involving the temporal bone following trauma, to assess ossicular injuries or dislocations and to assess complications arising from trauma.

\section{MATERIAL AND METHODS}

Study was done for one year on all patients who were referred to the Department of Radio diagnosis, B J Medical college and Sassoon General Hospitals, Pune 400001, for trauma to brain and ear with:

a) Ear bleed

b) Fluid coming out of ear

c) Swelling

d) Hearing loss whether temporary or permanent.

Written informed consent was obtained from all patients before taking for CT scan. In case of females in reproductive age group, caution was taken about excluding pregnancy by following 10 day rule and doing pelvic ultrasound, if required. The patients filled a consent form.

Exclusion criteria: 1. Extremely uncooperative patients due to associated head injuries. 2. Pregnant females or in females where the exact LMP was not known. They were scanned later for HRCT temporal bone if required.

Prospective analysis of $50 \mathrm{CT}$ scans was performed. All patients were studied on 64 slice CT scanner (Siemens) for injuries occurring to the temporal bone and its complications.

Scanning protocol: All patients were lying supine in the 
gantry. The images were obtained in a way to minimize exposure to the lens as far as possible. All scans were obtained in the helical mode. The coverage was from the arcuate eminence to the mastoid tip as is described in literature. Images were obtained in axial plane only. Thin reconstruction was performed in axial and coronal planes later. The parameters used were $120 \mathrm{kvp}, 250-320 \mathrm{mAs}$ depending on the age and $0.7 \mathrm{~mm}$ collimation. All images were read at $0.7 \mathrm{~mm}$ interval on axial and coronal sections. The images were reconstructed separately for right and left temporal bones using a bone filter algorithm. The FOV for axial used was $90-100 \mathrm{~mm}$ and for coronal was $180-200 \mathrm{~mm}$. The coronal images of both temporal bones was read together without any separation. Dose reduction techniques were used in paediatric cases as per the requirement. All images were read on dedicated workstations provided by Siemens. The analysis was carried out by 2 radiologists in the department. The observations were compared between the two radiologists before giving the diagnosis. Various postprocessing methods like multi-planar reconstruction (MPR), curved MPR, maximum intensity projection (MIP), surface shaded display (SSD) and volume rendering technique (VRT) were used to demonstate the fractures in a better way to clinicians.

Certain parameters were used to assess the quality of the CT scans obtained. The things seen were whether the singular canal is seen on CT or the middle and apical turns of cochlea were seen or not. The bone algorithm filter helps in creating good quality images of HRCT temporal bone. Also, a $0.7 \mathrm{~mm}$ reconstruction is much better than $1 \mathrm{~mm}$ reconstruction in assessing bony details about the temporal bone and to assess fractures or trauma in general. With thinner reconstructions, no partial volume artifacts are seen, which can lead to errors in diagnosis.

In patients in whom the entire brain scan was also performed for trauma, separate reconstructions were obtained for the temporal bone and a repeat $\mathrm{CT}$ was avoided to reduce radiation to the patient. If the reconstructions were unsatisfactory, then a separate thin CT of the temporal bone was obtained.

\section{STATISTICAL ANALYSIS}

The data was entered into Microsoft excel sheet and the descriptive statistics was performed. Bar diagram and other analysis was performed. Analysis was performed regards the kind of fractures, age and sex of the patients etc.

\section{RESULTS}

In our study, longitudinal fracture was more commonly seen. Transverse fracture was less common. Fractures were seen in 20 cases out of $50 \mathrm{CT}$ scans that we saw. Other patients had tympanic membrane ruptures or soft tissue swelling without any fracture or ossicular injury. 5 patients had ossicular injury. Out of the 20 cases of fractures, 13 were male patients and 7 were female patients. All patients with ossicular injuries were male patients. The commonest ossicular injury we found was malleoinducal dislocation. We saw one rare case of complete incudal dislocation in the external ear. Longitudinal fractures were seen in 15 cases and 5 cases had transverse fractures.

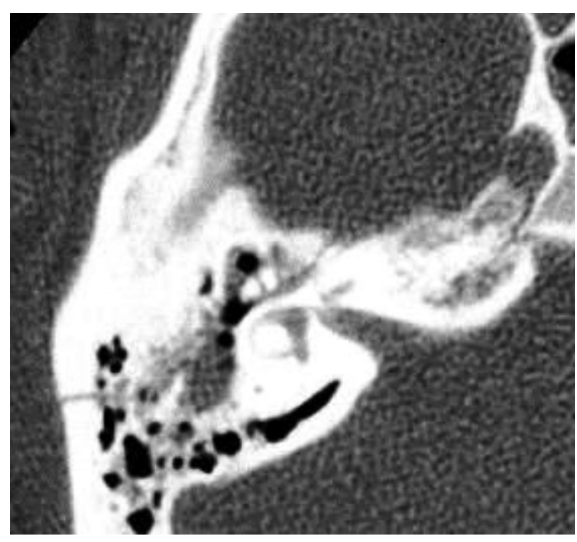

Figure-1: Longitudinal fracture with dislocation of of incudo-malleolar joint with disturbed ice-cone appearance.
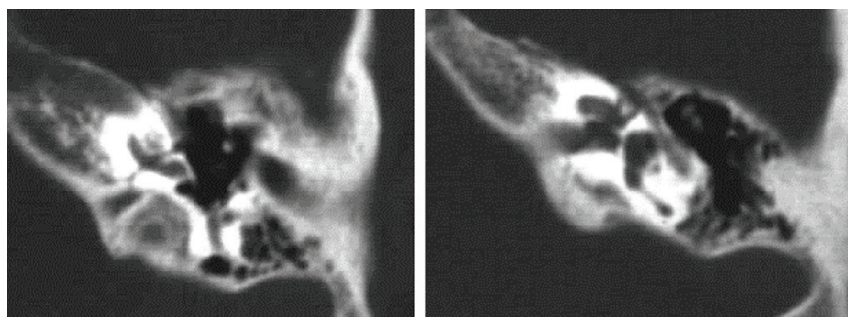

Figure-2a and b: Shows a transverse fractue of petrous bone crossing the turns of vestibule.
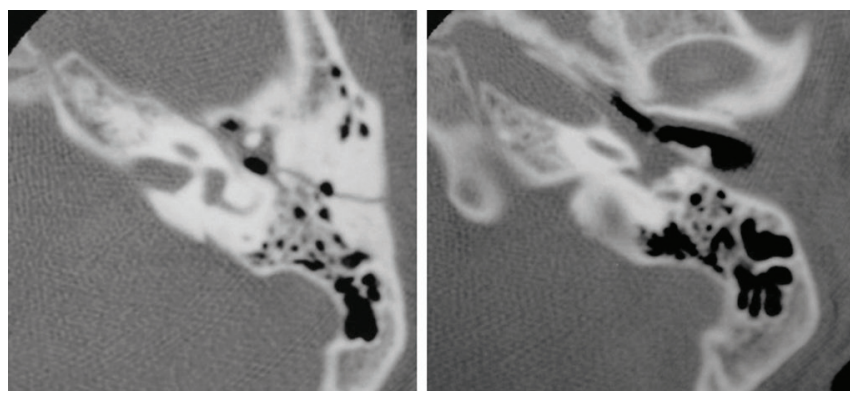

Figure-3: Longitudinal fracture with incus dislocation (extruded into external auditory meatus. (Ref 7))

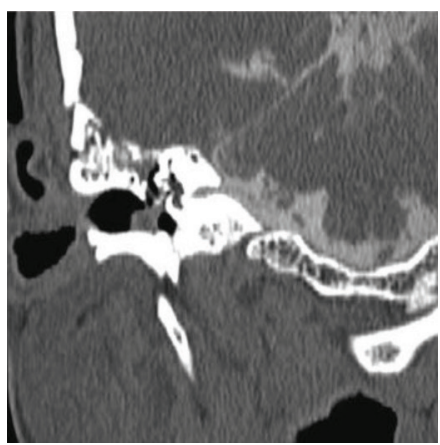

Figure-4: H/o head injury due to Otorrhoea due to small leak beneath defect in tegmen tympani

Figure 1 shows longitudinal fracture. The fracture line runs parallel to the long axis of the right temporal bone with disturbed ice-cream cone appearance of the malleus and incus.

Figure 2 shows transverse fracture of left temporal bone. The fracture line runs through the cochlea(a) and also extends upto the horizontal segment of left facial nerve.(b) 
25

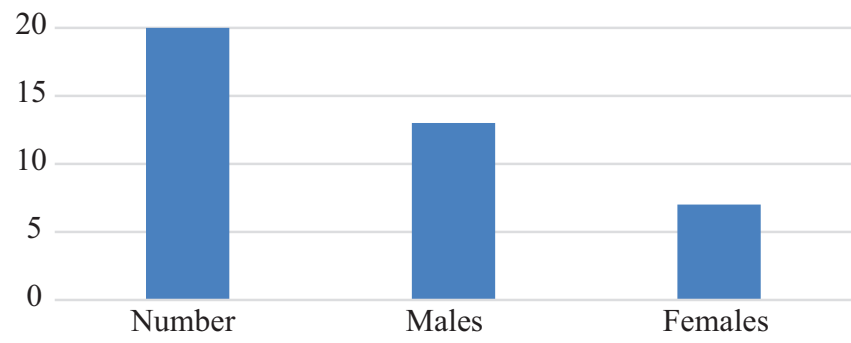

Figure-1: Bar diagram showing male and female distribution of fractures of temporal bone

Figure $3 \mathrm{a}$ and $\mathrm{b}$ show longitudinal fracture of left temporal bone with complete incudal dislocation. The incus lies in the external auditory canal.

Figure 4 shows complication of tegmen tympani fracture. The CSF was seen leaking in the right middle ear cavity on CT cisternography.

\begin{tabular}{|l|c|c|c|c|}
\hline $\begin{array}{l}\text { Total } \\
\text { number } \\
\text { of } \\
\text { patients }\end{array}$ & $\begin{array}{c}\text { No of } \\
\text { patients } \\
\text { with } \\
\text { fractures }\end{array}$ & $\begin{array}{c}\text { Longitudi- } \\
\text { nal fractures }\end{array}$ & $\begin{array}{l}\text { Transverse } \\
\text { fractures }\end{array}$ & $\begin{array}{c}\text { Ossicular } \\
\text { injuries }\end{array}$ \\
\hline 50 & 20 & 15 & 5 & 5 \\
\hline
\end{tabular}

\section{DISCUSSION}

In our study, the commonest fracture was longitudinal fracture of temporal bone. This is also described in literature. ${ }^{6}$ The complications of longitudinal fracture described are tympanic membrane rupture, ossicular injury and hemotympanum. Hemotympanum was seen in all the patients with fracture in our study. One patient had complete incudal dislocation with longitudinal fracture. This is uncommon but described in literature. ${ }^{4,5} \mathrm{~A}$ unique case of an ossicular chain injury was reported in a young man in literature. ${ }^{2,3}$ Despite the fact that the patient's incus was dislocated into the external auditory canal while remaining attached to the stapes, his hearing was not affected and remained nearly normal. Rarely incus has been reported to extend into vestibule, carotid canal or tympanic membrane.

Thomsen ${ }^{3}$ reported a material of 40 patients with diagnosis of dislocation of the incus is presented. 37 of these patients had either a history of cranial trauma, or information about previous simple mastoidectomy or paracentesis. The ossicles are deprived of their essential blood supply and yet nearly unharmed.

Nilesh Gupta, and Manish Meshram ${ }^{4}$ reported a rare case of dislocation of incus into the external acoustic canal following a fall from stairs with head injury. The most common form of ossicular dislocation after temporal bone trauma is separation of the joint connecting the incus to the stapes. The second most common is separation of the joint connecting the malleus to the incus. In our study, we found only malleoincudal dislocation. This could be due to a small cohort study. The other studies described in literature have a much larger cohort.

The incus is relatively vulnerable to traumatic dislocations (incudostapedial) on account of its weakly anchored position between the firmly attached malleus and stapes. After severe skull trauma also the incus may suffer dislocation. Malleoincudal dislocation may also occur. ${ }^{5,6}$ The incus may remain in the epitympanic recess, dislocate to the most inferior portion of the tympanic cavity or of the external ear canal, or even be destroyed.

Transverse fractures are further classified as otic capsule sparing and involving fractures. ${ }^{5,6}$ Out of the 5 transverse fractures we saw in the study, 3 had otic capsule involvement and 2 were otic capsule sparing fractures. The figure 2 shows fracture line involving the cochlea. These fractures can result in permanent sensorineural deafness in patients.

Complications of trauma: We had once case with tegmen tympani fracture. This patient started getting CSF otorrhoea. We performed CT cisternography in this patient. It showed leak of CSF in the middle ear. ${ }^{7}$ (Figure 4)

Other complications are also described in literature ossicular disruption-commonly incus and cholesteatoma and $7^{\text {th }}$ nerve paralysis. One of the patients with transverse fracture had $7^{\text {th }}$ nerve paralysis extending upto the horizontal portion of the nerve (figure 2b). We didn't see patient developing cholesteatoma. This could be due to lack of follow up of all the patients later on.

The other injuries are: malleoincudal joint dislocation, Stapediovestibular dislocation, Stapediovestibular dislocation, Labyrinthine fractures, dural fistulas, facial paralysis or injury to carotid canal. Tympanic membrane may or may not be perforated. The causes are external injury to temporal bone, injury by knitting needle, ear bud or pick. ${ }^{1}$

Treatment in any case is middle ear exploration and ossicular chain reconstruction. This surgery is essentially the same as tympanoplasty; however, there is no hole in the tympanic membrane to fix. Sometimes, the surgery can be performed though the ear canal rather than having to make an incision behind the ear.

\section{CONCLUSION}

Longitudinal fracture was more common than transverse fracture. This is also described in literature. Malleoincudal dislocation was seen in our study than other injuries. Only one case of complete incudal dislocation in the external auditory canal was seen.

Hemotympanum was seen in all patients with fracture of temporal bone. CSF otorrhoea was seen in one patient after we performed CT cisternography on the patient.

\section{REFERENCES}

1. Sertac Yetiser, Yusuf Hidir, Hakan Birkent. Traumatic ossicular dislocations: etiology and management. American Journal of Otolaryngology. Am J Otolaryngol 29(1):31-6.

2. Ana Maria Doffémond Costa; Juliana Oggioni Gaioti Caroline Laurita Batista Couto; Renata Lopes Furletti, et al. Temporal bone trauma and complications: computed tomography findings. Radiol Bras 2013;46(3);2:23-30.

3. Holzapfel AR, Chang CY, Pereira KD. Deguine C, Pulec JL. Ossicular chain dislocation with normal hearing. Ear Nose Throat J. 2005; 84(6):351-3.

4. Nilesh Gupta, Dr. Manish Meshram. Post-Traumatic dislocation of the incus into the external acoustic canal: 
a rare case report. Ear Nose Throat J. 1995; 74(12):800,

5. Julio O. Zayas, Yara Z. Feliciano, Celene R. Hadley, Angel A. Gomez, Jorge A. Vidal.Temporal bone trauma and role of MDCT in Emergency Department. Radio Graphics 2011; 31(1):1741-1755

6. Stewart C. Little, Bradley W. Kesser. Radiographic classification of temporal bone fractures. Otolaryngol Head Neck Surg. 2006;132(5):1300-1304.

7. Mukund D Rahalkar, Anand M Rahalkar, Vardhan S Joshi, Kailash V Sant, Kourabhi B Zade. Computed Tomography Cisternography for the Management of Cerebrospinal Fluid Fistulae. Otorhinolaryngology Clinics: An International Journal, 2017;9(3):1-127

Source of Support: Nil; Conflict of Interest: None

Submitted: 13-07-2019; Accepted: 12-08-2019; Published online: 04-09-2019 